\title{
State Fusion Estimation Based on Asynchronous Multirate Multisensor
}

\author{
Xiongjie Li \\ Department of Electronic and Information \\ Zhejiang Business Technology Institute \\ Ningbo 315012, China \\ e-mail: lixiongjie@tsinghua.org.cn
}

\author{
Paizhong Zhang \\ Department of Electronic and Information \\ Zhejiang Business Technology Institute \\ Ningbo 315012, China \\ e-mail: 731644838@qq.com
}

\begin{abstract}
On theory of multisensor state fusion estimation, more research is a single rate synchronization problem, however, it is the multirate asynchronous problem often encountered in practice. Therefore, research on the state fusion estimation of asynchronous multirate multisensor have more practice application value. In this paple, by expand the dimension of the system state and measurements and by dividing them into proper data blocks, the multirate asynchronous sampling system is formalized into a synchronous sampling system with single sampling rate, therefore, by use of Kalman filter and Carlson optimal data fusion criterion, the optimal state fusion estimation in the sense of linear minimum variance is achieved. The experiment of state fusion estimation on radar tracking shows that this algorithm is better than the result of directed Kalman filtering on smallest scale, the estimation error is less than single sensor Kalman filtering. The method can also be used for integrated navigation, signal processing, image processing and many fields.
\end{abstract}

Keywords-state fusion estimation; asynchronous; multirate; multisensor; Kalman filter

\section{INTRODUCTION}

Information fusion has been widely applied in many military and non-military fields because it has the advantages of improving the reliability and the stability, expanding the space and the time covering scope, and improving scale etc.

On theory of multisensor state fusion estimation, more research is a single rate synchronization, each of the sensors with the same sampling rate and synchronous sampling. However, it is the asynchronous problem often encountered in practice, the sensors with different sampling rate. Therefore, research on the state fusion estimation of asynchronous multirate multisensor have more practice application value.

In the 1989 annual meeting of IEEE control and decision, Willskyt first proposed the concept of multiscale system theory[1], Thus a good platform of multirate multisensor information fusion is provided. Subsequently, Hong proposed multiscale system and multi sensor information fusion theory[2], Wen etal presented multiscale estimation theory of dynamic systems [3], Cristi and Tummala introduced a multirate, multiresolution, recursive Kalman filter algorithm [4], Chen etal presented the multirate Kalman comprehensive filtering method[5], multirate filtering estimation algorithm[6] of Andrisani etal, asynchronous data fusion estimation[7] of Alouani etal, all these, have contributed to the research of asynchronous multirate multisensor information fusion.

In this paper, aimed at a multirate multisensor dynamic system, using the model of data block and expand dimension[8], the asynchronous multirate multisensor information fusion in the form into synchronous single rate information fusion, and the use of Kalman filtering and Carlson optimal data fusion criterion[9], achieve multisensor state fusion estimation. The experiment of state fusion estimation on radar tracking shows that this algorithm is better than the result of directed Kalman filtering on smallest scale, the estimation error is less than single sensor Kalman filtering.

The rest of this paper is organized as follows: Section 2 provides a scheme for the multisensor multirate asynchronous sampling. In Section 3, the asynchronous multirate multisensor state fusion estimation algorithm is presented. Section 4 gives some simulation experiment results, and Section 5 concludes the paper.

\section{MULTISENSOR MULTIRATE ASYNCHRONOUS SAMPLING}

\section{A. Problem formulation}

A multisensor linear dynamic system model with $N$ sensors can be described by

$$
\begin{gathered}
\boldsymbol{x}(N, k+1)=\boldsymbol{A}(N, k) \boldsymbol{x}(N, k)+\boldsymbol{w}(N, k) \\
\boldsymbol{z}(i, k)=\boldsymbol{C}(i, k) \boldsymbol{x}(i, k)+\boldsymbol{v}(i, k), \quad i=1,2, \Lambda, N
\end{gathered}
$$

The dynamic system is modeled at the highest sampling rate (the finest scale $\mathrm{N}$ ), where $\boldsymbol{x}(N, k) \in \boldsymbol{R}^{n \times 1}$ is the state variable at time $\mathrm{k}$ at the finest scale N. $\boldsymbol{A}(N, k) \in \boldsymbol{R}^{n \times n}$ is the system matrix. The modeling error $\boldsymbol{w}(N, k) \in \boldsymbol{R}^{n \times 1}$ is a white Gaussian noise, which satisfies

$$
\begin{gathered}
E\{\boldsymbol{w}(N, k)\}=0 \\
E\left\{\boldsymbol{w}(N, k) \boldsymbol{w}^{T}(N, l)\right\}=\boldsymbol{Q}(N) \boldsymbol{\delta}_{k l}
\end{gathered}
$$

There are $\mathrm{N}$ sequences of measurements (Eq. (2)) whose sampling rates decrease successively by any positive integers, i.e.

$$
S_{j+1}=n_{j} S_{j}, 1 \leq j \leq N-1
$$

where $n_{j}$ are known positive integers. $\boldsymbol{x}(i, k)$ is the kth state vector at scale $\mathrm{i}$, and $\boldsymbol{z}(i, k) \in \boldsymbol{R}^{q_{i} \times 1}\left(q_{i} \leq n\right)$ is the kth measurement observed by sensor $i$ with sampling rate $S_{i} . \boldsymbol{C}(i, k) \in \boldsymbol{R}^{q_{i} \times n}$ is the measurement matrix. The 
measurement error $\boldsymbol{v}(i, k) \in \boldsymbol{R}^{q_{i} \times 1}$ is supposed to be a white Gaussian noise, which satisfies

$$
\begin{gathered}
E\{\boldsymbol{v}(i, k)\}=0 \\
E\left\{\boldsymbol{v}(i, k) \boldsymbol{v}^{T}(j, l)\right\}=\boldsymbol{R}(i) \boldsymbol{\delta}_{i j} \boldsymbol{\delta}_{k l} \\
E\left\{\boldsymbol{v}(i, k) \boldsymbol{w}^{T}(N, l)\right\}=0, \quad k, l>0
\end{gathered}
$$

The initial value of the state vector $\boldsymbol{x}(N, 0)$ is a random vector with

$$
\begin{gathered}
E\{\boldsymbol{x}(N, 0)\}=\boldsymbol{x}_{0} \\
E\left\{\left[\boldsymbol{x}(N, 0)-\boldsymbol{x}_{0}\right]\left[\boldsymbol{x}(N, 0)-\boldsymbol{x}_{0}\right]^{T}\right\}=\boldsymbol{P}_{0}
\end{gathered}
$$

It is assumed that $\boldsymbol{x}(N, 0), \boldsymbol{w}(N, k)$ and $\boldsymbol{v}(i, k)$ are independent of each other. In (2), $i(1 \leq i \leq N)$ denote sensors as well as scales. Sensor $N$, which has the highest sampling rate, corresponds to the finest scale, $i=1$ corresponds to the coarsest scale, other sensor ( $i=2,3, \Lambda, N-1$ ) sampling rate is between 1 and $\mathrm{N}$, rising successively.

An example is depicted in Fig.1, where three scales are shown. One can see that the sampling rates of the sensors change from the highest ( scale $\mathrm{N}=3$ ) to the lowest (scale 1). It is assumed in Fig. 1 that the sampling rates have the ratio of $S_{3}: S_{2}=2: 1$ and $S_{2}: S_{1}=3: 1$, i.e. $n_{2}=2$ and $n_{1}=3$. From Fig .1, one can see that the sampling between different sensors can be asynchronous.

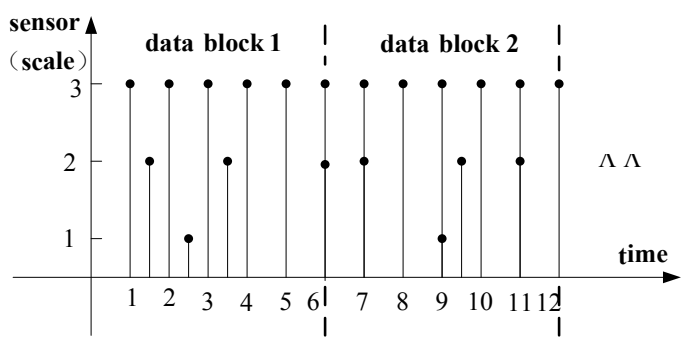

Figure 1. Schematic diagram of multisensor asynchronous sampling

If the sensor $\mathrm{N}$ sampling data into data block length is $n_{1} \cdot n_{2} \Lambda n_{N-1}$, then the length of data blocks in Fig. 1 is $n_{1} \cdot n_{2}=3 \times 2=6$. With the highest sampling rate of the $\mathrm{N}$ sensor is required uniform sampling, the sensor $i$ $(1 \leq i \leq N-1)$ can be non-uniform sampling, but in each data block within the same sampling frequency, i.e. different sensors with different sampling rate is asynchronous. How to fusion each sensor observation information, to obtain the optimal state estimation of the maximum sampling rate $\mathrm{N}$, we can use the method of data block model.

\section{B. Data block and dimension expand model}

According to the linear dynamic system with multirate multisensor asynchronous sampling, literature [8] presented the following data block and the dimension expand model.

Consider the type (1), (2) dynamic system with $\mathrm{N}$ sensor, if $\boldsymbol{x}(i, k)$ is obtained from the weighted average of adjacent several points in the finest scale, i.e. $\boldsymbol{x}(i, k)=\left(1 / M_{i}^{0}\right) \sum_{l=0}^{M_{i}^{\circ}-1} \boldsymbol{x}\left(N, M_{i}^{0} k-1\right), \tilde{M}_{i}=\prod_{j=i}^{N-1} n_{j}$ $(i=1,2, \Lambda, N-1)$, and where $M_{i}=\prod_{j=0}^{i-1} n_{j}, M=M_{N}$,
$\tilde{M}_{N} \underline{=} 1, n_{0} \triangleq 1$, then the data block and dimension expand model at scale $i(1 \leq i \leq N)$ can be written as

$$
\begin{gathered}
\boldsymbol{X}_{N}(k+1)=\boldsymbol{A}_{N}(k) \boldsymbol{X}_{N}(k)+\boldsymbol{W}_{N}(k) \\
\boldsymbol{Z}_{i}(k)=\boldsymbol{C}_{i}(k) \boldsymbol{X}_{N}(k)+\boldsymbol{V}_{i}(k)
\end{gathered}
$$

where

$$
\begin{aligned}
& \boldsymbol{X}_{N}(k)=\left[\begin{array}{c}
\boldsymbol{x}(N,(k-1) M+1) \\
\boldsymbol{x}(N,(k-1) M+2) \\
\mathrm{M} \\
\boldsymbol{x}(N, k M)
\end{array}\right] \\
& \boldsymbol{A}_{N}(k)=\left[\begin{array}{cccc}
0 & 0 & \mathrm{~L} & \boldsymbol{A}(N, k M) \\
0 & 0 & \mathrm{~L} & \boldsymbol{A}(N, k M+1) \boldsymbol{A}(N, k M) \\
\mathrm{M} & \mathrm{O} & \mathrm{M} \\
0 & 0 & \mathrm{~L} & \prod_{l=M-1}^{0} \boldsymbol{A}(N, k M+l)
\end{array}\right] \\
& \boldsymbol{Z}_{i}(k)=\left[\begin{array}{c}
\boldsymbol{z}\left(i,(k-1) M_{i}+1\right) \\
\boldsymbol{z}\left(i,(k-1) M_{i}+2\right) \\
\mathrm{M} \\
\boldsymbol{z}\left(i, k M_{i}\right)
\end{array}\right] \\
& \boldsymbol{C}_{i}(k)=\frac{1}{M_{i}^{0}} \operatorname{diag}\left\{\boldsymbol{C}\left(i,(k-1) M_{i}+1\right) \boldsymbol{I}_{M_{i}^{\circ}},\right. \\
& \left.\boldsymbol{C}\left(i,(k-1) M_{i}+2\right) \boldsymbol{I}_{M_{i}^{*}}, \mathrm{~L}, \boldsymbol{C}\left(i, k M_{i}\right) \boldsymbol{I}_{M_{i}^{q}}\right\}
\end{aligned}
$$

and where $I_{\mathscr{M}_{i}^{\circ}}=\left[\begin{array}{llll}I_{n} & I_{n} & \mathrm{~L} & I_{n}\end{array}\right]$ is a matrix with $n \times n \tilde{M}_{i}$ dimensions, which is composed of $\tilde{M}_{i}$ identity matrices with $n$ dimensions. $\boldsymbol{W}_{N}(k)$ and $\boldsymbol{V}_{i}(k)$ are zero-mean, white Gaussian noise sequences satisfying, the covariance respectively for $\boldsymbol{Q}_{N}(k)$ and $\boldsymbol{R}_{i}(k)$, i.e.

$$
\begin{gathered}
\boldsymbol{Q}_{N}(k)=\boldsymbol{B}_{N}(k) \operatorname{diag}\{\boldsymbol{Q}(N, k M), \boldsymbol{Q}(N, k M+1), \mathrm{L}, \\
\boldsymbol{Q}(N, k M+M-1)\} \boldsymbol{B}_{N}^{T}(k) \\
\boldsymbol{R}_{i}(k)=\operatorname{diag}\left\{\boldsymbol{R}\left(i,(k-1) M_{i}+1\right),\right. \\
\boldsymbol{B}_{N}(k)=\left[\begin{array}{cccc}
\left.\boldsymbol{R}\left(i,(k-1) M_{i}+2\right), \mathrm{L}, \boldsymbol{R}\left(i, k M_{i}\right)\right\} \\
\boldsymbol{I} & 0 & \mathrm{~L} & 0 \\
\boldsymbol{A}(N, k M+1) & \boldsymbol{I} & \mathrm{L} & 0 \\
\mathrm{~L} & & \mathrm{O} & \mathrm{M} \\
\prod_{l=M-1}^{1} \boldsymbol{A}(N, k M+l) & \prod_{l=M-1}^{2} \boldsymbol{A}(N, k M+l) & \mathrm{L} & \boldsymbol{I}
\end{array}\right]
\end{gathered}
$$

By dividing the data, including the state and the measurements etc., into data blocks, the asynchronous multirate sensor dynamic system (1) and (2) transform into (11) and (12) dynamic system of synchronous single rate sensor representation, as a result, the problem is simplified. Proof. see literature [8].

\section{STATE FUSION ESTIMATION ALGORITHM}

\section{A. Kalman filter on different scales}

The asynchronous multirate sensor dynamic system (1) and (2) transform into (11) and (12) synchronous single rate sensor dynamic system, then Kalman filter on different scales. The specific steps are as follows:

1) Dimension expansion: State variables are expanded dimension processing. According to the formula (13), $\boldsymbol{x}(N, k) \in \boldsymbol{R}^{n \times 1}$ will be converted into $\boldsymbol{X}_{N}(k) \in \boldsymbol{R}^{(M \times n) \times 1}$. 
2) calculation: According to equation (14), (15), (16), (17), (18), calculate $\boldsymbol{A}_{N}(k), \boldsymbol{Z}_{i}(k), \boldsymbol{C}_{i}(k), \boldsymbol{Q}_{N}(k), \boldsymbol{R}_{i}(k)$, where $i=1,2, \Lambda, N$.

3) Kalman filte: Kalman filtering is performed on the system model (11) and (12) of different observation scales $i$, Kalman recursive filtering algorithm is as follows

a) Initialization: Set the initial value $\hat{\boldsymbol{X}}_{N}(0 \mid 0)$ 、 $\boldsymbol{P}_{N}(0 \mid 0)$.

b) Forecast:

$$
\hat{\boldsymbol{X}}_{i, N}(k+1 \mid k)=\boldsymbol{A}_{N}(k) \hat{X}_{i, N}(k \mid k)
$$

c) Calculation of the residual sequence:

$$
\boldsymbol{\gamma}_{i}(k+1)=\boldsymbol{Z}_{i}(k+1)-\boldsymbol{C}_{i}(k) \hat{X}_{i, N}(k+1 \mid k)
$$
matrix:

d) Calculation of the prediction error covariance

$$
\boldsymbol{P}_{i, N}(k+1 \mid k)=\boldsymbol{A}_{N}(k) \boldsymbol{P}_{i, N}(k \mid k) \boldsymbol{A}_{N}^{T}(k)+\boldsymbol{Q}_{N}(k)
$$

e) Calculation of the gain matrix:

$$
\begin{aligned}
\boldsymbol{K}_{i}(k+1)= & \boldsymbol{P}_{i, N}(k+1 \mid k) \boldsymbol{C}_{i}^{T}(k) \\
& {\left[\boldsymbol{C}_{i}(k) \boldsymbol{P}_{i, N}(k+1 \mid k) \boldsymbol{C}_{i}^{T}(k)+\boldsymbol{R}_{i}(k)\right]^{-1} }
\end{aligned}
$$

$f$ ) Computation of the state estimation error covariance matrix:

$$
\begin{aligned}
\boldsymbol{P}_{i, N}(k+1 \mid k+1) & =\boldsymbol{P}_{i, N}(k+1 \mid k) \\
& -\boldsymbol{K}_{i}(k+1) \boldsymbol{C}_{i}(k) \boldsymbol{P}_{i, N}(k+1 \mid k)
\end{aligned}
$$

g) To update:

$$
\hat{\boldsymbol{X}}_{i, N}(k+1 \mid k+1)=\hat{\boldsymbol{X}}_{i, N}(k+1 \mid k)+\boldsymbol{K}_{i}(k+1) \gamma_{i}(k+1)
$$

Finally, make $k=k+1$, repeat the above steps.

\section{B. Optimal state fusion criterion}

In 1990 Carlson proposed an optimal state fusion criterion[9], For $i=1,2, \Lambda, N$, suppose that $\hat{\boldsymbol{X}}_{i, N}(k \mid k)$ and $\boldsymbol{P}_{i, N}(k \mid k)$ are the state estimates and the estimation error covariance matrices of $X_{N}(k)$ by Kalman filtering based on model (11) and (12) respectively, which are independent of each other, then $X_{N}(k)$ optimal fused estimate in the sense of linear minimum covariance is given by

$$
\hat{\boldsymbol{X}}_{N}(k \mid k)=\sum_{i=1}^{N} \alpha_{i, k} \hat{\boldsymbol{X}}_{i, N}(k \mid k)
$$

where

$$
\alpha_{i, k}=\left(\sum_{j=1}^{N} \boldsymbol{P}_{j, N}^{-1}(k \mid k)\right)^{-1} \boldsymbol{P}_{i, N}^{-1}(k \mid k)
$$

and the corresponding estimation error covariance matrix

$$
\boldsymbol{P}_{N}(k \mid k)=\left(\sum_{i=1}^{N} \boldsymbol{P}_{i, N}^{-1}(k \mid k)\right)^{-1}
$$

In addition, it can be proof

$$
\boldsymbol{P}_{N}(k \mid k) \leq \boldsymbol{P}_{i, N}(k \mid k)(i=1,2, \mathrm{~L}, N)
$$

Fig .2 shows the flowchart of the state fusion estimation algorithm based on model (11) and (12).

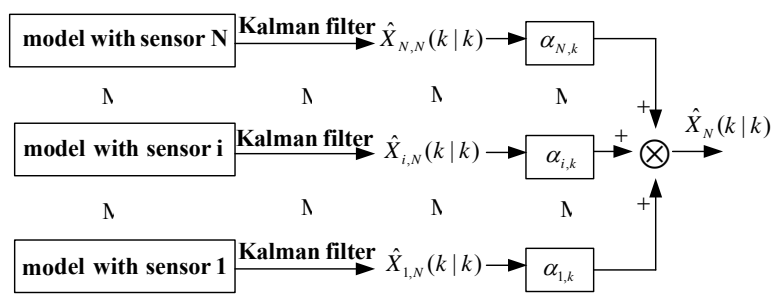

Figure 2. Flowchart of the state fusion estimation algorithm

\section{STATE FUSION ESTIMATION SIMULATION EXPERIMENT}

Constant acceleration model of radar tracking with 3 sensors is considered[10], the discrete state equation as follows

$$
\begin{gathered}
\boldsymbol{x}(t+1)=\left[\begin{array}{ccc}
1 & T & T^{2} / 2 \\
0 & 1 & T \\
0 & 0 & 1
\end{array}\right] \boldsymbol{x}(t)+\boldsymbol{w}(t) \\
\boldsymbol{z}(i, t)=\boldsymbol{C}(i, t) \boldsymbol{x}(i, t)+\boldsymbol{v}(i, t), i=1,2,3
\end{gathered}
$$

where $T$ is the sampling period. The state vector $\boldsymbol{x}(t)=\left[\begin{array}{lll}s(t) & (t)\end{array}\right]^{T}$, where $s(t) 、(t)$ and are the position, velocity and acceleration, respectively, of the target at time $t T \cdot \boldsymbol{z}(i, t) \quad(i=1,2,3)$ are the measurements of three sensors, which observe the position, the velocity and acceleration, respectively, i.e. $\boldsymbol{C}(1)=\left[\begin{array}{lll}1 & 0 & 0\end{array}\right], \boldsymbol{C}(2)=\left[\begin{array}{lll}0 & 1 & 0\end{array}\right], \boldsymbol{C}(3)=\left[\begin{array}{lll}0 & 0 & 1\end{array}\right] \cdot v(i, t)$ are zero-mean white Gaussian noises with varances $R(i)$, and are independent of white Gaussian noises $w(t)$ with zero-mean and varances $Q$. Our objective now is to obtain the best estimate of the target $\boldsymbol{x}(t)$ by fusing the information from three sensors. In the simulation, setting $T=0.01 s, Q=0.1, \quad R(3)=2, \quad R(2)=1, \quad R(1)=0.1$. The values are $\boldsymbol{x}(0)=\left[\begin{array}{lll}0 & 0.1 & 10\end{array}\right]$ and $\boldsymbol{P}_{0}=0.1 \cdot \boldsymbol{I}_{3}$. The sampling ratio between the different sensors are $S_{3}: S_{2}=2: 1$ and $S_{2}: S_{1}=3: 1$, as shown in Fig.1.

The fine scale sensor sampling 600, the mean statistics of 10 times Monte Carlo simulation estimation error absolute value are shown in Table 1 . We can see from Table I , comprehensive estimation error of 3 sensor fusion is the smallest.

TABLE I . Comparison between the mean of the estimation error

\begin{tabular}{ccccc}
\multicolumn{5}{c}{ absolute value } \\
\hline & $\begin{array}{c}\text { sensor } \\
1\end{array}$ & $\begin{array}{c}\text { sensor } \\
2\end{array}$ & $\begin{array}{c}\text { sensor } \\
3\end{array}$ & 3 sensor fusion \\
\hline $\begin{array}{c}s(t) \\
\text { estimation error }\end{array}$ & 0.1464 & 2.1867 & 6.1786 & 0.1437 \\
\hline $\begin{array}{c}\&(t) \\
\text { estimation error }\end{array}$ & 0.9672 & 0.1403 & 1.5071 & 0.1383 \\
\hline $\begin{array}{c}\text { (ft }) \\
\text { estimation error }\end{array}$ & 0.7791 & 0.7379 & 0.1095 & 0.1141 \\
\hline $\begin{array}{c}\text { comprehensive } \\
\text { estimation error }\end{array}$ & 0.6309 & 1.0216 & 2.5984 & 0.1320 \\
\hline
\end{tabular}


The first sensor state estimation curve is shown Fig .3, the second sensor state estimation curve is shown in Fig .4, the third sensor state estimation curve is shown in Fig .5, the 3 sensor state fusion estimation curve is shown in Fig .6. If you carefully observe these state estimation curve, then the single sensor state estimation curve are not very good, Fig .3 on the velocity and acceleration tracking is not very good, Fig .4 on the position and acceleration tracking is not very good, Fig .5 on the position and speed tracking is not very good, only Fig .6 of the position, velocity and acceleration tracking are good. Thus, compared with the single sensor Kalman filter result, 3 sensor fusion estimation values are more close to the target signal, so as to prove the validity of this algorithm.
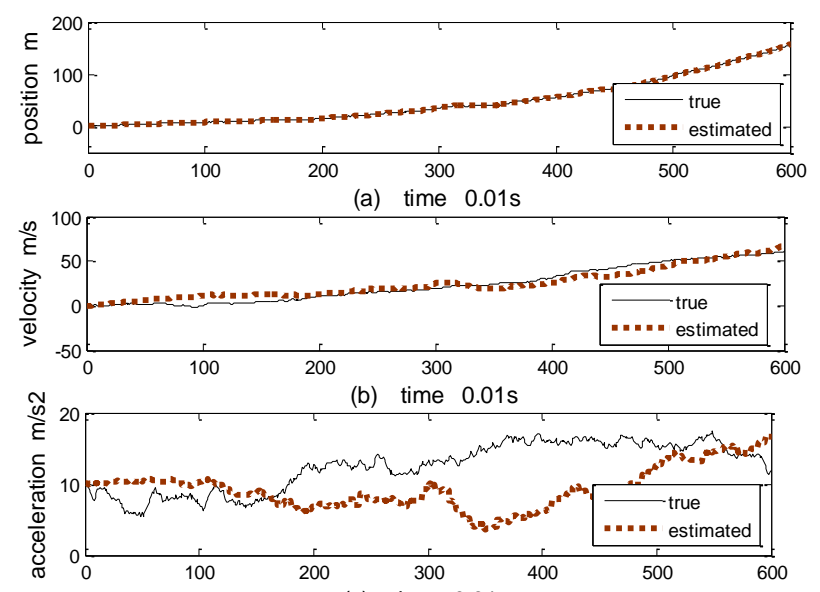

(c) time $0.01 \mathrm{~s}$

Figure 3. The first sensor state estimation curve
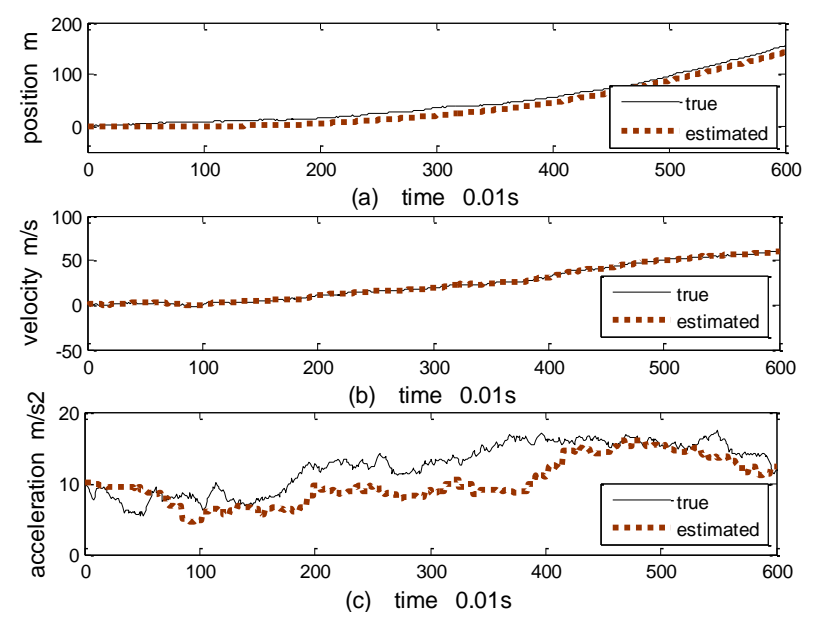

Figure 4. The second sensor state estimation curve

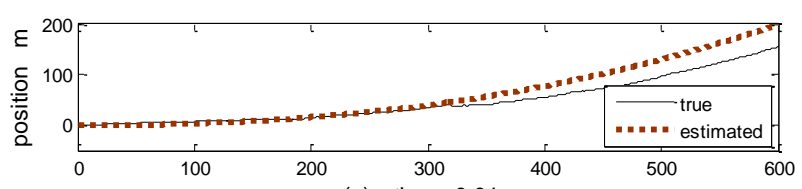

(a) time $0.01 \mathrm{~s}$
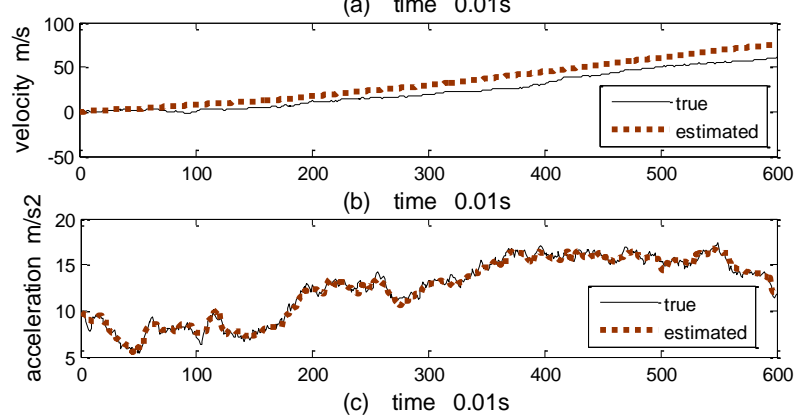

Figure 5. The third sensor state estimation curve

\section{CONCLUSIONS}

Information fusion technology can improve system reliability and stability, state fusion estimation is one of the research focus. This paper, in the known state equation ( most fine scale) and the observation equation (various scale) under the condition of, application of data block and dimension expand model, application of Kalman filter and the optimal state fusion criterion, realize the asynchronous multirate sensor state fusion estimation. Simulation results show the tracking radar system, obtained the 3 sensor information fusion state estimation error is smaller than the estimation error of using any single sensor, so this method is very effective. The method can also be used for integrated navigation, signal processing, image processing, fault detection, fault tolerant control and many fields.

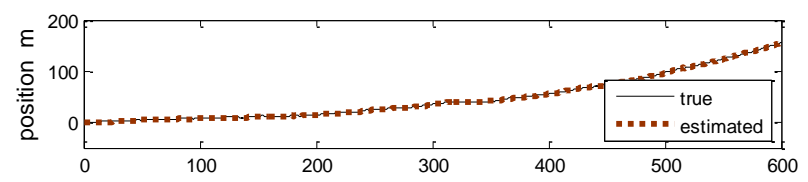

(a) time $0.01 \mathrm{~s}$
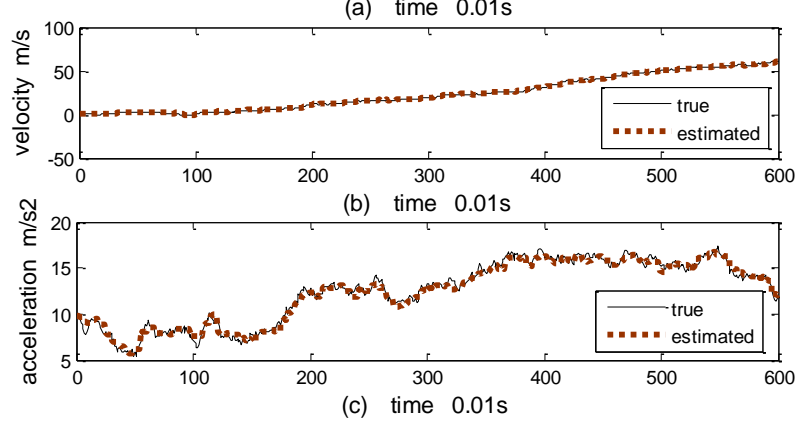

Figure 6 . The 3 sensor fusion state estimation curve

\section{ACKNOWLEDGMENT}

This work was supported by Guangdong Province petrochemical equipment fault diagnosis key laboratory open fund (grant No. 201332) 


\section{REFERENCES}

[1] Chou K. C., Willsky, A. S., Benveniste, A., Basseville, M. Recursive and iterative estimation algorithms for multiresolution stochastic processes, In Proceedings of the 28th IEEE Conference on Decision and Control, Fampa, Florida, 1989, 2: 1184 -1189.

[2] Hong, L. Multi-resolutional distributed filtering, IEEE Transactions on Automatic Control, 1994, 39 (4): 853-856.

[3] Wen C.L., Zhou D.H. Multiscale Estimation Theory and Application, Publishing House of Tsinghua University, Beijing, 2002(in Chinese).

[4] Cristi, R., Tummala, M. Multirate, multiresolution, recursive Kalman filter, Signal Processing, 2000, 80 (9): 1945-1958.

[5] Chen B. S., Lin C. W., Chen Y. L. Optimal signal reconstruction in noisy filter bank systems: multirate Kalman synthesis filtering approach, IEEE Transactions on signal Processing, 1995, 43(11): 2496-2500.
[6] Andrisani D., Gau C.F. Estimation Using a Multirate Filter. IEEE Transactions on Automatic Control, 1987, 32(7): 653-656.

[7] Alouani A. T., Rice T.R. On optimal synchronous and asynchronous track fusion [J].Optical Engineering, 1998, 37(2):427-433.

[8] Yan L.P., Liu B.S., Zhou D.H. The modeling and estimation of asynchronous multirate multisensor dynamic systems, Aerospace Science and Technology, 2006, 10(1):63-71

[9] Carlson, N. A. Federated square root filter for decentralized parallel processors[J], IEEE Transactions on Aerospace and Electronic Systems, 1990, 26 (3): 517-525.

[10] Sun S. L. Multi-sensor optimal information fusion Kalman filters with applications[J], Aerospace Science and Technology, 2004, 8(1): 57-62. 